\title{
A Longitudinal Analysis of Early Lesion Growth in Presymptomatic Patients with Cerebral Adrenoleukodystrophy
}

\author{
(D)E.J. Mallack, (D) G. Askin, (DS. van de Stadt, (DP.A. Caruso, (DP.L. Musolino, (D) M. Engelen, (DS.N. Niogi, and (D)F.S. Eichler
}

\begin{abstract}
BACKGROUND AND PURPOSE: Cerebral adrenoleukodystrophy is a devastating neurological disorder caused by mutations in the $A B C D I$ gene. Our aim was to model and compare the growth of early cerebral lesions from longitudinal MRIs obtained in presymptomatic patients with progressive and arrested cerebral adrenoleukodystrophy using quantitative MR imaging-based lesion volumetry.

MATERIALS AND METHODS: We retrospectively quantified and modeled the longitudinal growth of early cerebral lesions from 174 MRIs obtained from 36 presymptomatic male patients with cerebral adrenoleukodystrophy. Lesions were manually segmented using subject-specific lesion-intensity thresholding. Volumes were calculated and plotted across time. Lesion velocity and acceleration were calculated between sequentially paired and triplet MRIs, respectively. Linear mixed-effects models were used to assess differences in growth parameters between progressive and arrested phenotypes.
\end{abstract}

RESULTS: The median patient age was 7.4 years (range, 3.9-37.0 years). Early-stage cerebral disease progression was inversely correlated with age $(\rho=-0.6631, P<.001)$, early lesions can grow while appearing radiographically stable, lesions undergo sustained acceleration in progressive cerebral adrenoleukodystrophy $\left(\beta=0.10 \mathrm{~mL} / \mathrm{month}^{2}\left[95 \% \mathrm{Cl}, 0.05-0.14 \mathrm{~mL} / \mathrm{month}{ }^{2}\right], P<.001\right)$, and growth trajectories diverge between phenotypes in the presymptomatic time period.

CONCLUSIONS: Measuring the volumetric changes in newly developing cerebral lesions across time can distinguish cerebral adrenoleukodystrophy phenotypes before symptom onset. When factored into the overall clinical presentation of a patient with a new brain lesion, quantitative MR imaging-based lesion volumetry may aid in the accurate prediction of patients eligible for therapy.

ABBREVIATIONS: CALD = cerebral adrenoleukodystrophy; $\mathrm{HSCT}=$ hematopoietic stem cell transplantation; $\mathrm{LS}=$ Loes score; $\mathrm{t}_{0}=$ time-zero

$\mathbf{X}$ -linked adrenoleukodystrophy is a devastating neurologic disorder caused by mutations in the $A B C D 1$ gene, which lead to an accumulation of very long chain fatty acids in plasma and tissue. ${ }^{1}$ Multiple phenotypes emerge with no genotypephenotype relationship having been established. ${ }^{2}$ Most patients will develop cerebral adrenoleukodystrophy (CALD), with the highest incidence occurring in childhood. ${ }^{3}$ Lesions most often occur in the splenium $(60 \%-80 \%)$ or genu $(10 \%-15 \%)$ of the

Received January 29, 2021; accepted after revision May 18.

From the Departments of Neurology (E.J.M., P.L.M, F.S.E.) and Radiology (P.A.C.), Harvard Medical School, Massachusetts General Hospital, Boston, Massachusetts; Department of Pediatrics (E.J.M.), Division of Child Neurology, Weill Cornell Medicine, NewYork-Presbyterian Hospital, New York, New York; Department of Population Health Sciences (G.A.), Division of Biostatistics, and Department of Radiology (S.N.N.), Weill Cornell Medicine, New York, New York; and Amsterdam Leukodystrophy Center (S.v.d.S, M.E.), Department of Pediatric Neurology, Emma Children's Hospital, Amsterdam University Medical Center, Amsterdam, the Netherland.

S.N. Niogi and F.S. Eichler are senior authors.

Statistical analysis was performed by Eric Mallack, MD, 175 Cambridge Street, Suite 340, Boston, MA 02114, and Gulce Askin, MPH, 402 E. 67th St, Level C2, New York, NY 10065. corpus callosum and spread confluently into the surrounding subcortical white matter. ${ }^{4,5}$ More than $80 \%$ of children with CALD will experience inflammatory demyelination, "progressive CALD," followed by rapid neurodegeneration and death without treatment in 2-3 years. ${ }^{6,7}$ Conversely, $15 \%-20 \%$ of children and most adults will undergo spontaneous arrest of disease, "arrested CALD" without evidence of brain inflammation and are ineligible for hematopoietic stem cell transplantation (HSCT). ${ }^{8}$

HSCT is most successful when initiated in the window before the onset of neurologic symptoms. ${ }^{7,9-11}$ However, the presymptomatic window is narrow, ${ }^{12,13}$ and only a minority of patients with

All phases of this study were supported by the National Institute of Health and the Leblang Charitable Foundation. Dr Musolino is supported by a $\mathrm{K} 08$ (1K08NS094683-01), and Dr Mallack, by a K12 (5 K12 NS066274), both from the National Institute of Neurological Disorders and Stroke.

Please address correspondence to Eric Mallack, MD, 525 East 68th St, Box 91, New York, NY 10065; e-mail: ejm9009@med.cornell.edu

- Indicates open access to non-subscribers at www.ajnr.org

Indicates article with online supplemental data.

http://dx.doi.org/10.3174/ajnr.A7250 
progressive CALD are identified in time for HSCT. ${ }^{7}$ HSCT itself is associated with significant toxicity, morbidity, and mortality, the latter 2 of which increase with disease burden. ${ }^{7,10}$ Early identification and treatment of CALD is a challenge. ${ }^{7,12}$ Most important, the addition of adrenoleukodystrophy to the Recommended Uniform Screening Program has provided a new opportunity to alter the natural history of the disease by monitoring for CALD from birth. ${ }^{14}$

The MR imaging score, or Loes score (LS), is the standard metric for brain demyelination in CALD. ${ }^{15}$ Previous work has quantified the increase in the MR imaging score per year stratified by lesion distribution. ${ }^{5}$ However, the LS is not specific nor predictive of progression early in CALD and underestimates the true burden of cerebral disease. ${ }^{4,16}$ There is an explicitly stated need for early biomarkers to aid in the accurate prediction of patients with CALD appropriate for therapy., ${ }^{7,17}$

Few prior studies focused on the kinetics of 3D CALD lesion growth in presymptomatic patients. We recently demonstrated that lesion growth is rapid when lesions are small, the growth rate is inversely correlated with age, and older patients tend to stabilize and undergo spontaneous arrest of disease. ${ }^{4}$ The primary aim of this study was to model the growth of early cerebral lesions from longitudinal MRIs obtained in presymptomatic patients with CALD using lesion volumetry, a quantitative MR imaging approach. We hypothesized that the trajectories of lesion growth diverge between the progressive and arrested phenotypes before symptom onset. Hence tracking the growth of de novo cerebral lesions may enable the early diagnosis of patients with CALD appropriate for HSCT and thereby facilitate clinical decisionmaking around this progressive disease.

\section{MATERIALS AND METHODS \\ Standard Protocol Approvals, Registrations, and Consents}

Patient data were retrospectively reviewed and de-identified, and storage was encrypted and password-protected. Due to anonymization, consent was waived. This study was approved by each institution's ethics/institutional review board.

\section{Subjects}

We performed a retrospective review of patients with CALD evaluated at 3 institutions (Massachusetts General Hospital, Amsterdam University Medical Center, and Weill Cornell Medicine) to select cases for study inclusion. The inclusion criteria were the following: 1) confirmed diagnosis of adrenoleukodystrophy by genetic testing ( $A B C D 1$ gene mutation) or high levels of plasma very long chain fatty acids; ${ }^{18}$ 2) early-stage $\mathrm{CALD}^{7,19}$ defined as no cerebral symptoms as defined by the neurologic functional score (range, 0-25) and the LS on first MR imaging with abnormal findings (ie, MR imaging at diagnosis) between 0.5 and nine; 3) $\geq 2$ available brain MRIs per subject; 4) at least 1 axial T2-weighted sequence per MR imaging; 5) MRIs obtained in the pretreatment time period (no history of hematopoietic stem cell or gene therapy); and 6) pattern 1 or 2 lesion distributions only (genu or splenium of corpus callosum).

Patients were divided into 2 study cohorts by clinical diagnosis: progressive CALD versus arrested CALD. Diagnosis of arrested CALD was defined by $\geq 2$ consecutive MRIs spanning a minimum of 6 months with no increase in LS and no contrast enhancement. ${ }^{8}$ Analyses were limited to callosal lesions to compare growth parameters accurately between subjects over similar neuroanatomic boundaries. Included patients could have a neurologic functional score of $>0$ if the symptoms were attributed to adrenomyeloneuropathy. Treatment with Lorenzo Oil did not exclude patients from participation in the study. ${ }^{1} \mathrm{MR}$ imaging surveillance every 6 months between 3 and 10 years of age, published by the New York State Newborn Screening workgroup, was followed. ${ }^{14}$ Otherwise, MR imaging and clinical follow-up were completed per clinical determination.

\section{MR Imaging Parameters}

MR imaging studies of the brain in the Amsterdam University Medical Center cohort were performed on a 3T MR imaging unit (Ingenia; Phillips Healthcare). MR imaging studies in the Weill Cornell Medicine cohort were performed on a 3T MR imaging unit (MR750; GE Healthcare). One-hundred forty-two of the 150 MR imaging studies in the Massachusetts General Hospital cohort were performed on a 3T MR imaging unit (Tim Trio [Siemens] until 2017, Prisma 2017 to present, [Siemens]). Eight studies obtained before 2015 were performed on 1.5T MR imaging units (Signa HDxt, GE Healthcare; or Ingenia, Philips Healthcare). Each subject was scanned longitudinally according to their site's standard clinical protocol. Axial conventional T2, conventional FLAIR, or sampling perfection with applicationoptimized contrasts by using different flip angle evolution (SPACE, Siemens) FLAIR volumetric sequences were analyzed. Parameter ranges were the following: $\mathrm{TR}=5000-6500 \mathrm{~ms}, \mathrm{TE}=$ $100-393 \mathrm{~ms}$, frequency $=192-512$, phase $=75 \%-100 \%$, section thickness $=1-5 \mathrm{~mm}$, gap $=0-1 \mathrm{~mm}, \mathrm{FOV}=180-220 \times 180-$ $220 \mathrm{~mm}$.

\section{MR Imaging Analysis}

Loes Score. Loes scores were assigned by neuroradiologists (P.A.C., A.L., K.B.) or neurologists (F.S.E., P.L.M, M.E.) with a minimum of 10 years of experience in cerebral adrenoleukodystrophy. Reviewers evaluated the images according to the Loes scoring system for abnormal signal hyperintensity or atrophy involving specific brain structures known to be involved in CALD. ${ }^{15}$

Lesion Pattern. The lesions were subdivided into 5 patterns according to their primary anatomic distribution as previously published: ${ }^{5}$ 1) parieto-occipital lobe white matter and splenium of the corpus callosum; 2) frontal lobe white matter and genu of the corpus callosum; 3) frontopontine or corticospinal projection fibers; 4) cerebellar white matter; and 5) simultaneous parietooccipital and frontal white matter involvement. Only patients in whom the MR imaging showed at least either pattern 1 or pattern 2 lesions were included in this study. Pattern 3 lesions were included only if they were continuous with a frontal or parieto-occipital lesion.

Lesion Volume Calculation. 3D Slicer software (https://www. slicer.org/) was used to create 3D label maps of the lesion by thresholding the label intensity to match the lesion intensity on the source axial T2, conventional FLAIR, or SPACE FLAIR sequence. ${ }^{20,21}$ Lesions were then manually segmented section-bysection according to the subject-specific threshold value. Lesion 
volumes were automatically calculated from the compiled section-by-section lesion segmentations (ie, area of the segmentation $\times$ section thickness) and are reported in milliliters $(\mathrm{mL})$.

Interrater Reliability. To assess the interrater reliability of lesion segmentation between 2 raters (E.J.M. and A.L.) on the dataset, we calculated the intraclass correlation coefficient using a 2-way random-effects model with a consistency definition.

\section{Statistical Analyses}

Descriptive Statistics. All continuous variables are reported as median and range. Discrete variables are summarized as frequency (\%). The Wilcoxon rank sum test was used to calculate differences in median values among skewed samples. Two-tailed $P$ values $<$ .05 were considered statistically significant. SPSS Statistics (Version 25 ; IBM) was used to perform this section of the analyses.

Population-Level Analysis of Cerebral Lesion Growth. Raw lesion volumes versus age were plotted. Lesion velocity was defined as a change in lesion volume between 2 sequential MRIs divided by the difference in months between scans and is reported in milliliters per month (mL/month). Lesion acceleration was defined as the difference in lesion velocities between the 2 scans divided by the time interval between scans and is reported in milliliters squared per month $\left(\mathrm{mL} / \mathrm{month}^{2}\right)$. The Spearman rank correlation coefficient $(\rho)$ was calculated to quantify the relationship between patient age and lesion velocity. Similarly, $\rho$ was calculated to quantify the correlation between patient age and lesion acceleration.

Longitudinal Analysis of Individual Cerebral Lesion Growth. Time-zero $\left(t_{0}\right)$ was defined as the date of first abnormal MR imaging. Fold change was calculated as the ratio of lesion volume on the nth MR imaging at $t_{n}$ over the initial volume at $t_{0}$. Fold change in lesion volume for each patient was then plotted from $t_{0}$. Similarly, lesion velocity was plotted for each patient from $t_{0}$.

Lesion Growth Curve Modeling. The trajectory of each dependent variable was visualized using the nonparametric local-weighted regression curve with 95\% confidence intervals and a smoothing parameter $(\alpha)$ of 0.75 . Linear mixed-effects models were used to assess the difference in fold change and lesion velocity between the progressive and arrested phenotypes. Individual differences in baseline lesion volume and number/intervals of follow-up scans were addressed by assuming random intercepts for each patient. The model was fit with the main effects for phenotype group and time as well as an interaction term between phenotype and time:

$$
\begin{gathered}
\mathcal{Y}_{i j}=\beta_{0}+\beta_{1} \text { group }_{i}+\beta_{2} \text { time }_{i j}+\beta_{3} \text { time }_{i j} \text { group }_{i}+b_{i}+\varepsilon_{i j}, \\
\text { Group }\left\{\begin{array}{c}
1=\text { progressive } \\
0=\text { arrested }
\end{array}\right.
\end{gathered}
$$

$\mathcal{Y}_{i j}$ progressive $=\beta_{0}+\beta_{1}$ group $_{i}+\left(\beta_{2}+\beta_{3}\right)$ time $_{i j}+b_{i}+\varepsilon_{i j}$,

$$
\mathcal{Y}_{i j} \text { arrested }=\beta_{0}+\beta_{2} \text { time }_{i j}+b_{i}+\varepsilon_{i j},
$$

where

$$
\begin{aligned}
& \varepsilon \sim N\left(0, \sigma^{2}\right), \\
& b_{i} \sim N\left(0, \sigma_{b}^{2}\right) .
\end{aligned}
$$

The regression coefficient $(\beta),(95 \% \mathrm{CI})$, and $P$ value are reported for each parameter estimate from the linear mixed effect (LME) models.

Restricted Data Set Analysis To understand the growth of small lesions from the earliest time points, we recalculated the nonparametric local-weighted regression curves and linear mixed-effects model analyses from a subset of the data meeting more restricted criteria: LS $\leq 2$ on the first MRI with abnormal findings. ${ }^{13}$ To illustrate lesion growth across time, we plotted raw volumes in months from $t_{0}$. This section of the analysis was performed by a biostatistician using $\mathrm{R}$ statistical and computing software, Version 3.5.3 (http://www.r-project.org/).

\section{Data Availability}

Following publication, any data not published within this article will be anonymized and shared by request from any qualified investigator.

\section{RESULTS \\ Clinical Characteristics}

Thirty-six patients met the inclusion criteria, resulting in 174 scans available for analysis. The total LS range across all MRIs with abnormal findings was $0.5-7.5$. At the time of first MR imaging with abnormal findings, patients had a median age of 89.1 months (range, 47.2-449 months), with a median MR imaging LS of 1 (range, 0.5-7.5, Online Supplemental Data). Twenty-three patients were diagnosed with progressive CALD, accounting for 101 MRIs in the study. The median age at first MR imaging with abnormal findings was 71.8 months (range, 47.2-137.5 months), with an initial LS of 1 (range, 1-7). Thirteen patients underwent spontaneous arrest of cerebral disease, accounting for the remaining 73 MRIs. The median age at first MR imaging with abnormal findings was 185.6 months (range, 96.3-449 months), with an initial LS of 3 (range, 0.5-7.5). Patients with arrested CALD were older at the time of diagnosis (15.5 versus 6.0 years of age, $P<.001$ ).

A total of 23 patients met the more restricted criteria (LS $\leq 2$ at first MR imaging with abnormal findings), resulting in 125 MRIs eligible for analysis. The LS range was 0.5-6. Nineteen patients with progressive CALD were identified, accounting for 90 MRIs. They were 69.5 months of age (range, 47.2-137.5 months) at diagnosis and had an initial LS of 1 (range, 1-2). Four of the patients with arrested CALD were identified, accounting for the remaining 35 MRIs. They were 136.3 months of age (range, 96.3-185.6 months) and had an initial LS of 1 (range, 0.52). Patients with arrested CALD were older at the time of diagnosis (11.4 versus 5.8 years of age, $P=.003$ ).

\section{Lesion Segmentation}

The intraclass correlation coefficient between lesion segmentations produced by 2 raters (E.J.M. and A.L.) on 39 MRIs from the data set was 0.874 (95\% CI, 0.772-0.932; $P<.001)$, indicating a 

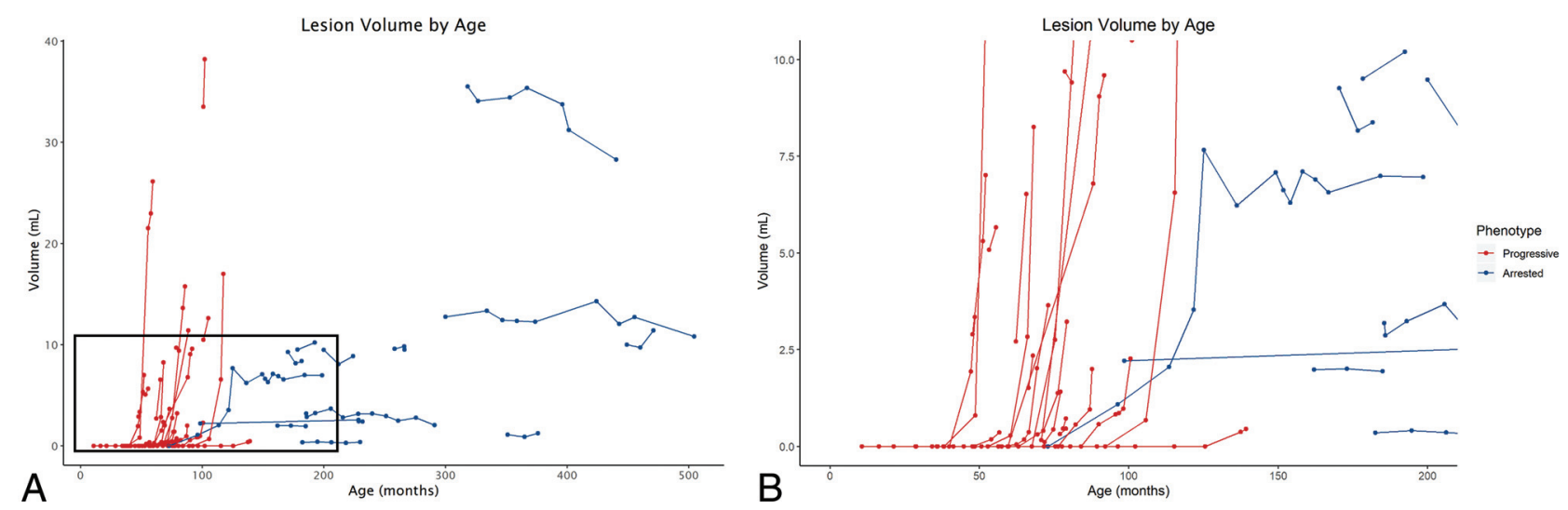

FIG 1. A, Patient-specific lesion trajectories of each cohort plotted against patient age. $B$, Zoomed view of outlined area in $1 A$ ): $0-18$ years of age on the $\mathrm{x}$-axis and 0 - to $10-\mathrm{mL}$ lesion volume on the $\mathrm{y}$-axis. Note the exponential growth trajectories for patients with progressive CALD.
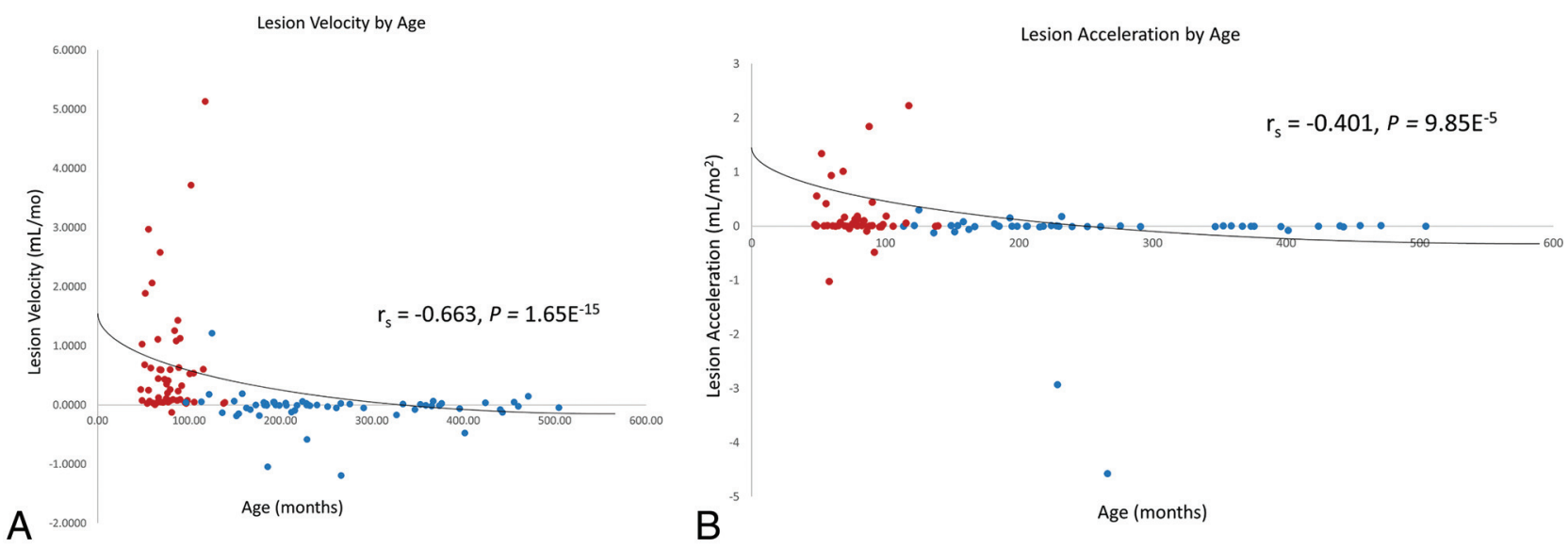

FIG 2. $A$, Lesion growth velocities over sequential MR imaging pairs and $B$, acceleration over sequential MR imaging triplets, stratified by age in patients with progressive CALD (red) and arrested CALD (blue). Presymptomatic lesion progression is inversely correlated with age $(P<.001)$.

significant, good-to-excellent degree of reliability between segmentations produced by different raters.

\section{Disease Progression is Inversely Correlated with Age}

Patient-specific lesion volumes were plotted across time against patient age in months (Fig 1). There was a moderate-to-strong negative correlation between patient age and lesion velocity $(n=112, \rho=-0.6631, P<.001$, Fig $2 A)$. Accordingly, lesion acceleration inversely correlated with patient age $(n=89, \rho=$ $-0.4010, P<.001$, Fig $2 B)$.

\section{The Loes Score is Insensitive to Early Lesion Growth}

There was a very strong correlation between lesion volume and LS ( $n=132, \rho=0.8086, P<.001)$. The median volume of a lesion with an LS of 1 was $0.51 \mathrm{~mL}$ (interquartile range, $0.36-1.46$ $\mathrm{mL}$; range, $0.05-8.26 \mathrm{~mL}$ ). The median volume of a lesion with an LS of 2 was $3.18 \mathrm{~mL}$ (interquartile range, $2.45-3.67 \mathrm{~mL}$; range, $0.68-11.41 \mathrm{~mL}$ ). The median fold change on longitudinal MRIs with an LS of 1 (ie, $\Delta \mathrm{LS}=0)$ was $1.83(n=16$; interquartile range, 1.47-5.71; range, 1.11-29.14). Similarly, the median rate of lesion growth on longitudinal MRIs with an LS of 1 was $0.08 \mathrm{~mL}$ / month $(n=30$; interquartile range, $0.05-0.25 \mathrm{~mL} / \mathrm{month}$; range, 0.01-2.58 mL/month; Fig 3).

\section{Lesions in Progressive CALD Undergo Early, Sustained Acceleration}

The 2-way interaction term between phenotype and time was significant for the rate of lesion growth (Table). For every 1 month, lesion velocity increased by $0.10 \mathrm{~mL} / \mathrm{month}$ in patients with progressive CALD ( $\beta=0.10$ [95\% CI 0.05-0.14], $P<.001$ ). There was a marginally larger effect in the restricted cohort $(\beta=0.11$ [95\% CI, 0.06-0.15], $P<.001$ ). The rate of lesion growth in patients with progressive CALD increased with time, consistent with exponential growth kinetics.

\section{Lesion Growth Trajectories Diverge between Phenotypes before the Onset of Cerebral Symptoms}

The 2-way interaction term between phenotype and time was significant for lesion growth measured by fold change (Table). Lesion volume increases by a factor of 2.5 from baseline every month in patients with progressive CALD (fold change $\beta=2.49$ [95\% CI, 2.10-2.89], $P<.001)$. This model is limited to MRIs of asymptomatic patients, with a median initial LS of 1 (range, 1-7), and an LS range of 1-7 on subsequent MRIs (Fig 4).

The results of the phenotype and time interaction from the restricted cohort were similar to those of the full cohort (Table): There 


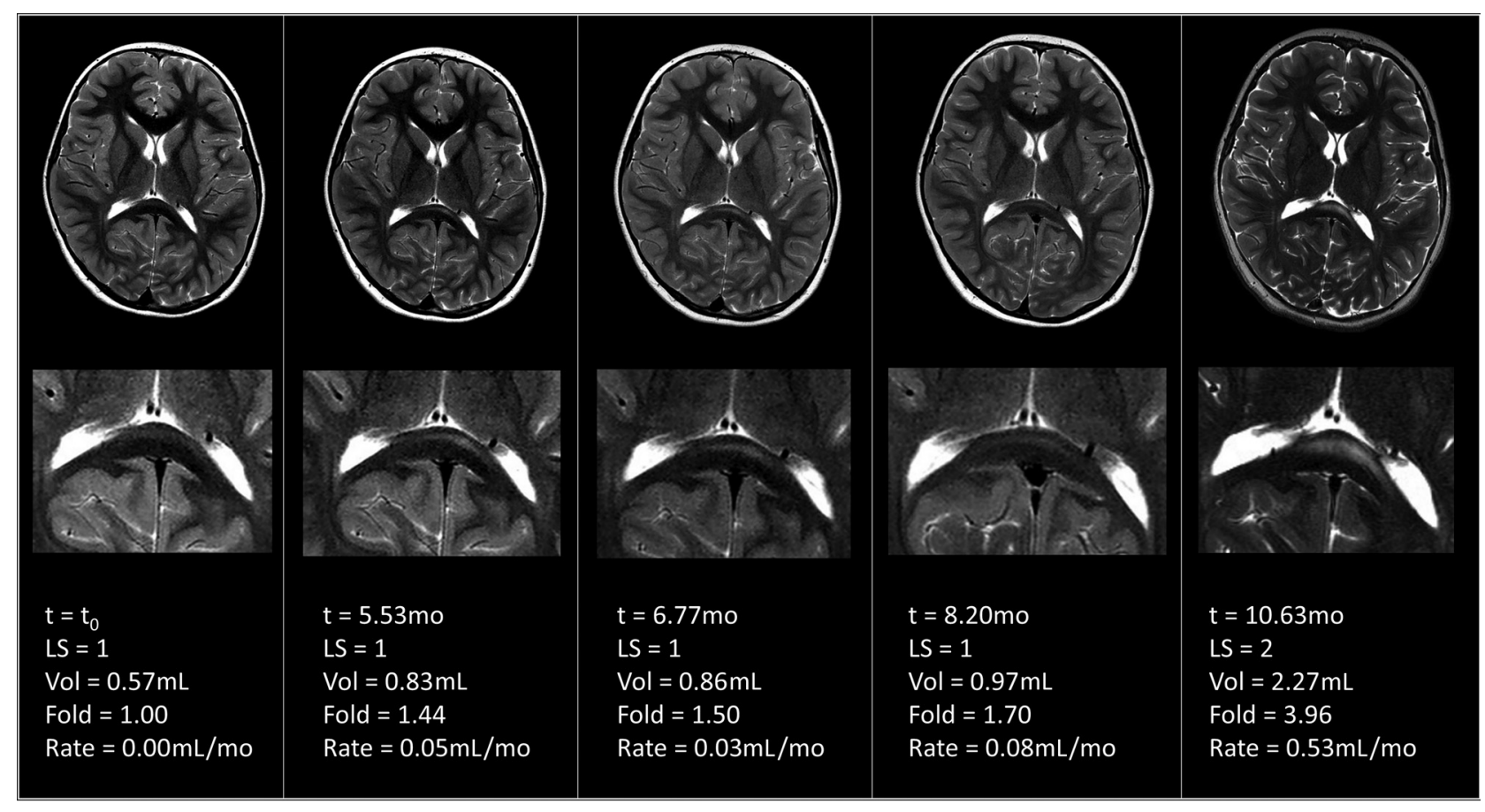

FIG 3. Serial MRIs demonstrating accelerated lesion growth without a change in the LS in patient 11.

Estimates produced by the linear mixed-effects models for fold change and lesion velocity in the full and restricted cohorts

\begin{tabular}{|c|c|c|c|c|c|c|}
\hline & \multicolumn{3}{|c|}{ Fold Change } & \multicolumn{3}{|c|}{ Lesion Velocity } \\
\hline & Estimate & $95 \% \mathrm{Cl}$ & $P$ & Estimate & $95 \% \mathrm{Cl}$ & $P$ \\
\hline \multicolumn{7}{|l|}{ Full cohort } \\
\hline Time & 0.01 & $-0.03-0.04$ & .768 & 0.00 & $-0.00-0.00$ & .989 \\
\hline Phenotype (progressive) & -3.00 & $-6.94-0.94$ & .142 & 0.340 & $-0.10-0.77$ & .136 \\
\hline Time*phenotype (progressive) & 2.49 & $2.10-2.89$ & $<.001$ & 0.100 & $0.05-0.14$ & $<.001$ \\
\hline \multicolumn{7}{|l|}{ Restricted cohort } \\
\hline Time & 0.01 & $-0.06-0.08$ & .752 & 0.00 & $-0.01-0.01$ & .899 \\
\hline Phenotype (progressive) & -3.43 & $-11.08-4.21$ & .386 & 0.15 & $-0.47-0.78$ & .632 \\
\hline Time* ${ }^{\star}$ henotype (progressive) & 2.54 & $2.04-3.04$ & $<.001$ & 0.11 & $0.06-0.15$ & $<.001$ \\
\hline
\end{tabular}

Note:-Asterisk indicates the interaction between phenotype and time in the linear mixed effects model.

was a marginally larger effect for fold change in lesion volume across time ( $\beta=2.54$ [95\% CI, 2.04-3.04], $P<.001)$. This model is limited to MRIs of asymptomatic patients with a median initial LS of 1 (range, 1-2) and an LS range of 1-6 on subsequent MRIs (Fig 4).

In both linear mixed-effects models, the main effect term for phenotype was not significant, indicating that the 2 groups did not differ at onset. This is expected because baseline MRIs with abnormal findings have a common corrected value for both phenotypes (ie, fold change $=1$, lesion velocity $=0 \mathrm{~mL} / \mathrm{month}$ ). The median rate of lesion growth directly following the first MR imaging with abnormal findings in the progressive group was significantly faster than that in the arrested group $(0.448 \mathrm{~mL} / \mathrm{month}$ versus $0.003 \mathrm{~mL} / \mathrm{month}, P=.001$ ).

To approximate real-time Newborn Screening MR imaging surveillance of early CALD, we plotted raw volumes from $t_{0}$ in the restricted cohort of patients (Fig 5).

\section{DISCUSSION}

Our study aimed to explore cerebral lesion growth as a potential early biomarker for progressive CALD. The implementation of the adrenoleukodystrophy Newborn Screening has provided the opportunity to monitor for new cerebral lesions from birth. ${ }^{14,22}$ The early, accurate diagnosis of progressive disease is paramount because HSCT is most successful when initiated in the window before the onset of neurologic symptoms. ${ }^{7,9,10,23}$ This may be equally important for other treatment approaches as well; a recent trial of autologous hematopoietic stem cell gene therapy has produced encouraging results in boys with early CALD. ${ }^{19}$ Because symptoms accelerate with time in patients with progressive CALD, ${ }^{3,5,6}$ we hypothesized that disease acceleration could be radiographically captured before symptom onset. We completed the primary aim by modeling the $3 \mathrm{D}$ expansion of early cerebral lesions from longitudinal MRIs obtained in presymptomatic patients with progressive and arrested CALD. Our results demonstrate that disease progression is inversely correlated with age, early lesions can grow while appearing radiographically stable, lesions undergo sustained acceleration in early-stage progressive CALD, and growth trajectories diverge between phenotypes in the presymptomatic time period. 

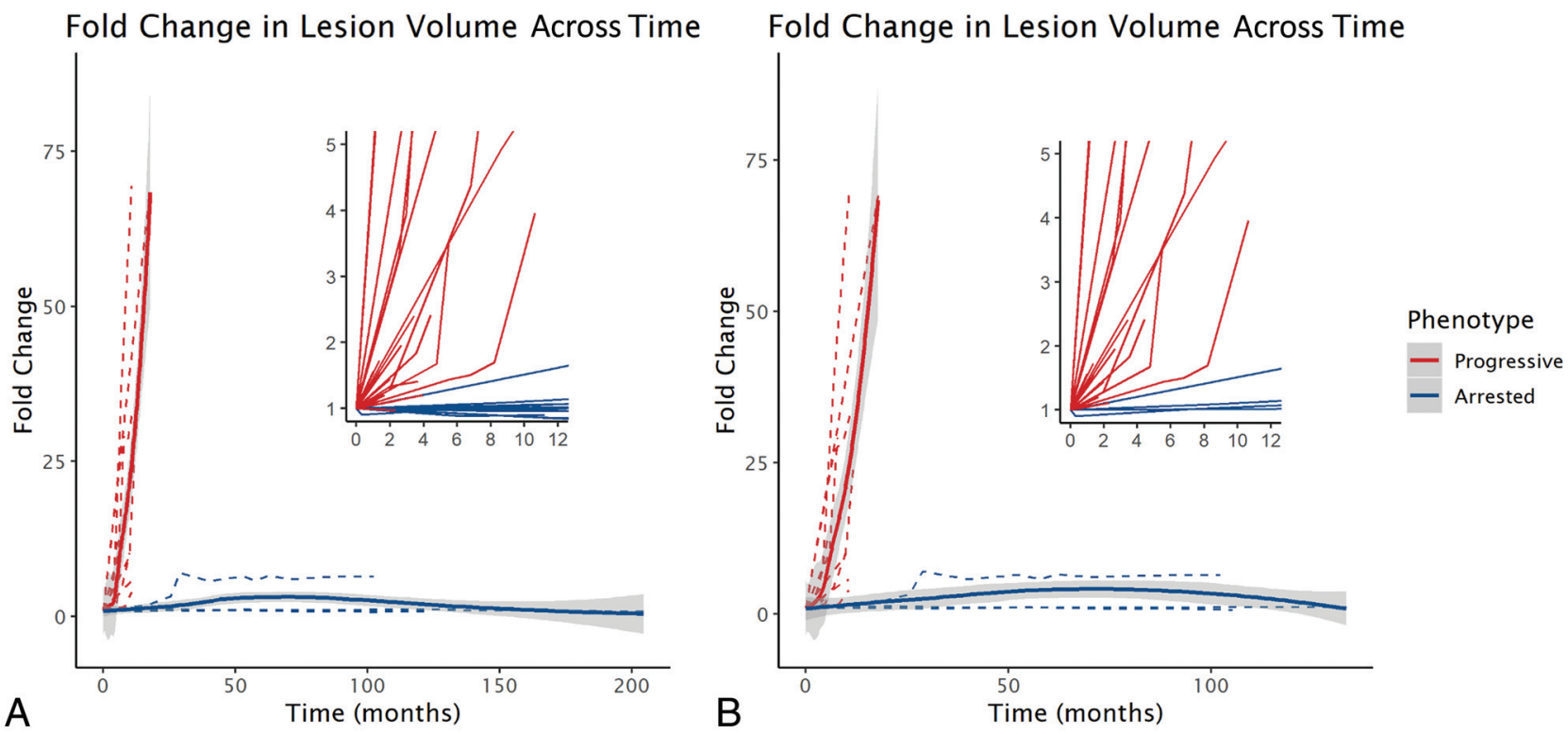

FIG 4. $A$, Lesion growth trajectories from the time of the first abnormal MR imaging finding in presymptomatic patients with progressive $(n=23$, red) versus arrested ( $n=13$, blue) CALD. Lesion volume increased $2.5 \times$ per month (fold change $\beta=2.49$ [95\% Cl, 2.10-2.89], $P<.001$ ) in the patients with progressive CALD. $B$, In the cohort of patients with an initial LS $\leq 2$ (restricted cohort; $n=19$ progressive, $n=4$ arrested), lesion growth was similar $(\beta=2.54$ [95\% Cl, 2.04-3.04], $P<.001)$ for the patients with progressive CALD.

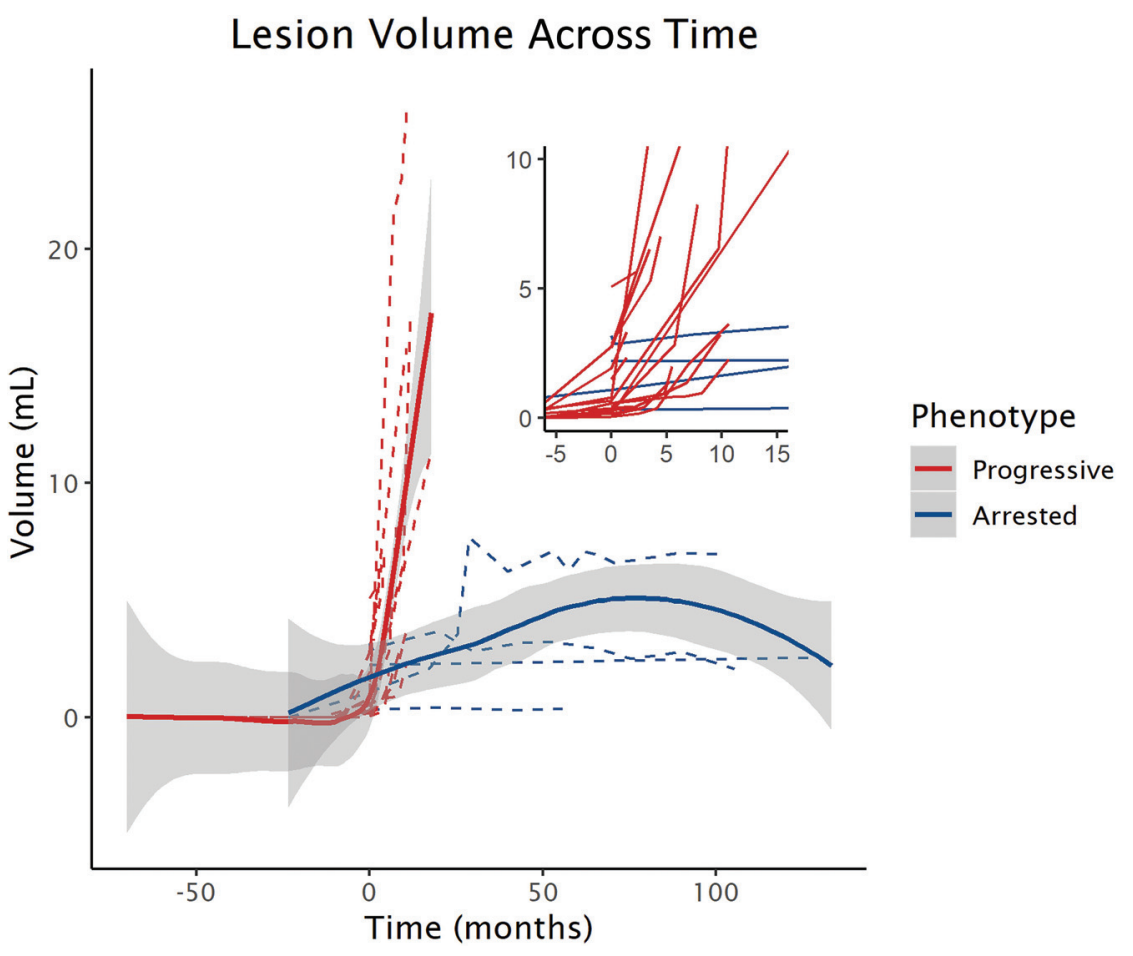

FIG 5. Nonparametric local-weighted regression curves demonstrating the early lesion growth trajectories between phenotypes measured by raw lesion volume across time in the cohort of patients with an initial LS $\leq 2$.

To further understand lesion expansion from the earliest time points, thus approximating what may be found by Newborn Screening MR imaging surveillance, we repeated the analysis on the subset of longitudinal MRIs that had an LS $\leq 2$ at diagnosis. In this restricted cohort, patients with both progressive and arrested CALD were diagnosed at a younger age with smaller lesions. Again, all measures of growth differed between phenotypes, indicating that trajectories diverge very early in disease and may be detected by calculating lesion velocity and fold change from the MR imaging following diagnosis.

Suspicion for progressive CALD should be highest in younger patients whose lesions follow an exponential growth curve. Our recent work demonstrated the inverse relationship between rates of demyelinating lesion growth and age in a cohort of asymptomatic patients with CALD. ${ }^{4}$ The current study validates our previous results in a larger, international cohort. Biologically, our findings are consistent with the timing of dynamic myelination into late adolescence and adulthood ${ }^{24}$ and the suggestion that following transplantation, more developed white matter tracts in adolescence protect against further neurocognitive decline compared with children with CALD undergoing the procedure. ${ }^{17}$ Stated another way, younger children with younger myelin may be more vulnerable to cerebral disease progression than older patients with more developed myelin.

The proposed model has identified features of early lesion growth that have the potential to inform the timely selection of patients appropriate for rescue therapy. This feature is pertinent 
to recent observations that altered cerebral microvascular perfusion in perilesional normal-appearing white matter precedes lesion progression. ${ }^{16}$ Early patient identification would help mitigate risk and maximize neurologic outcomes, decrease the number of MRIs required to identify the correct patients for treatment, reduce patient exposures to anesthesia and contrast agents, and widen the presymptomatic treatment window. Patients treated expeditiously with an LS $\leq 2$ have demonstrated superior posttransplantation neurocognitive and neuropsychiatric outcomes and less posttransplantation disease progression on MRI. ${ }^{13}$ Lesion volumetry may also prove to be a more sensitive method to monitor for disease activity posttreatment. In terms of feasibility, the biomarker itself is based on the acquisition of standard T2-weighted images and, therefore, makes the analysis paradigm possible across all platforms. Conceptually, an analysis of growth kinetics can be applied to other leukodystrophies, as it has been in other white matter diseases. ${ }^{25,26}$

Longitudinal imaging within subjects provides strength to the analysis. The statistical analyses accounted for the correlation between repeat patient measures, thus allowing each patient to act as an internal control for his or her lesion-specific changes across time. Sequential pair and triplet data points are required to calculate lesion growth velocity and acceleration, respectively, which improve the accuracy of the study conclusions. However, important limitations apply. First, we analyzed retrospective data and are limited by when subjects came to medical attention. The result is lesion onset (the transition from a normal MR imaging finding with a $L S=0$ to an abnormal MR imaging finding with a LS $\geq 0.5$ ) is not available for most of the arrested CALD cohort. This issue limits the comparison with the patients with progressive CALD for whom lesion onset was factored into the model. Only a standardized, prospective imaging surveillance program will reveal the true growth trajectory and behavior of cerebral adrenoleukodystrophy lesions across time. Second, there were an unequal number of scans per patient and unequal time between repeat measures. This intra- and intersubject heterogeneity precluded the use of more preferable methods of longitudinal mathematic modeling, including fitting more complex, nonlinear models. Third, the number of subjects, specifically in the arrested cohort, is limited. As with many rare diseases, the generalizability of the study conclusions is limited due to the number of available cases. Fourth, while most scans were sequentially obtained on the same MR imaging scanner for each subject, 5 subjects had their 1-2 MRIs obtained on a $1.5 \mathrm{~T}$ unit. This may have introduced variability in the interpretation of disease onset and the degree of white matter involvement. If present, the variability is small: Sicotte et $\mathrm{al}^{27}$ demonstrated a $10 \%$ average difference in total white matter lesion volume between $1.5 \mathrm{~T}$ and $3 \mathrm{~T}$ scanners. A similar effect may have been introduced by performing some measurements on the axial T2-weighted sequence versus 3D T2 FLAIR sequences. Fifth, our analysis did not include isolated pattern 3,4 , or 5 lesions because they are exceedingly rare in this age group. ${ }^{5}$ Sixth, manual lesion segmentations are time- and resource-intensive, making integration into clinical workflow difficult. Adaptation of pre-existing volume measurement methodologies or automated deep learning approaches to lesion segmentation may support workflow integration. ${ }^{28,29}$ Finally, the proposed models require validation before entertaining their use in clinical practice. While our models provide insight into early lesion behavior, we emphasize caution when interpreting the results in the context of clinical practice. If validated, our results lay the foundation for diagnostic criteria aimed at identifying early-stage progressive CALD in a fashion similar to that of other progressive neurologic diseases. ${ }^{30}$

\section{CONCLUSIONS}

The addition of adrenoleukodystrophy to Newborn Screening has provided clinicians a novel, presymptomatic approach to disease. Understanding early lesion evolution is paramount for the accurate, early diagnosis of patients with progressive CALD. Our study indicates that measuring the volumetric changes in newly developing cerebral lesions across time can distinguish CALD phenotypes before symptom onset. When factored into the overall clinical presentation of a patient with a new brain lesion, our method may aid in the accurate prediction of patients eligible for therapy.

\section{ACKNOWLEDGMENTS}

We are grateful to Afonso Liberato, $\mathrm{MD}$, for providing lesion segmentations on the data set; Wouter van Ballegoij, MD, and Karen Buch, MD, for providing additional help scoring patient MR imaging scans; Arne Lauer, MD for providing guidance on the data set and analysis; and Jerry W. Harper for editing the manuscript.

Disclosures: Eric J. Mallack-RELATED: Grant: National Institutes of Health/ National Institute of Neurological Disorders and Stroke K12 Neurological Sciences Academic Development Award.* Patricia L. Musolino-RELATED: Grant: National Institute of Neurological Disorders and Stroke, Comments: K08.*. Marc Engelen-UNRELATED: Consultancy: Minoryx Therapeutics, Autobahn, bluebird bio, Comments: research collaboration with pharmaceutical companies developing drugs for X-linked adrenoleukodystrophy*; Grants/Grants Pending: Vidi Grant, Netherlands Organization for Scientific Research, Comments: grant for natural history study in adrenoleukodystrophy; Payment for Development of Educational Presentations: cerebral adrenoleukodystrophy education video on Medscape, Comments: commissioned by bluebird bio. Sumit N. NiogiRELATED: Grant: K12 National Institutes of Health Award, Comments: The study was funded, in part, by a K12 National Institutes of Health Award to Weill Cornell Medicine with Dr Mallack as the Principal Investigator of the grant. I received no support through this grant mechanism.* Florian S. EichlerUNRELATED: Consultancy: SwanBio Therapeutics; Patents (Planned, Pending Or Issued): adenoassociated virus serotype-mediated $A B C D I$ gene correction; Payment for Development of Educational Presentations: PRIME medical education company, Comments: for live webinars on adrenoleukodystrophy; OTHER RELATIONSHIPS: Principal Investigator of the adrenoleukodystrophy gene therapy sponsored by bluebird bio, site Principal Investigator of the trial MIN102 sponsored by Minoryx Therapeutics. *Money paid to the institution.

\section{REFERENCES}

1. Moser HW. Adrenoleukodystrophy: phenotype, genetics, pathogenesis and therapy. Brain 1997;120:1485-1508 CrossRef

2. Huffnagel IC, Laheji FK, Aziz-Bose R, et al. The natural history of adrenal insufficiency in X-linked adrenoleukodystrophy: an international collaboration. J Clin Endocrinol Metab 2019;104:118-26 CrossRef Medline

3. Moser HW, Loes DJ, Melhem ER, et al. X-linked adrenoleukodystrophy: overview and prognosis as a function of age and brain magnetic resonance imaging abnormality-a study involving 372 patients. Neuropediatrics 2000;31:227-39 CrossRef Medline 
4. Liberato AP, Mallack EJ, Aziz-Bose R, et al. MRI brain lesions in asymptomatic boys with $\mathrm{X}$-linked adrenoleukodystrophy. Neurology 2019;92:e1698-1708 CrossRef Medline

5. Loes DJ, Fatemi A, Melhem ER, et al. Analysis of MRI patterns aids prediction of progression in $\mathrm{X}$-linked adrenoleukodystrophy. Neurology 2003;61:369-74 CrossRef Medline

6. Melhem ER, Loes DJ, Georgiades CS, et al. X-linked adrenoleukodystrophy: the role of contrast-enhanced MR imaging in predicting disease progression. AJNR Am J Neuroradiol 2000;21:839-44 Medline

7. Raymond GV, Aubourg P, Paker A, et al. Survival and functional outcomes in boys with cerebral adrenoleukodystrophy with and without hematopoietic stem cell transplantation. Biol Blood Marrow Transplant 2019;25:538-48 CrossRef Medline

8. Mallack EJ, van de Stadt S, Caruso PA, et al. Clinical and radiographic course of arrested cerebral adrenoleukodystrophy. Neurology 2020;94: e2499-2507 CrossRef Medline

9. Peters C, Charnas LR, DeFor T, et al. Cerebral X-linked adrenoleukodystrophy: the University of Minnesota hematopoietic cell transplantation experience from 1991 to 2004. Blood 2004;104:2153 CrossRef

10. Miller WP, Rothman SM, Nascene D, et al. Outcomes after allogeneic hematopoietic cell transplantation for childhood cerebral adrenoleukodystrophy: the largest single-institution cohort report. Blood 2011;118:1971-78 CrossRef Medline

11. Miller W, Bjoraker K, Charnas L, et al. Hematopoietic cell transplantation (HCT) for adrenoleukodystrophy: ten-year experience with 62 patients. Ann Neurol 2010;68:S121 CrossRef

12. Mahmood A, Raymond GV, Dubey $P$, et al. Survival analysis of haematopoietic cell transplantation for childhood cerebral X-linked adrenoleukodystrophy: a comparison study. Lancet Neurol 2007;6:687-92 CrossRef Medline

13. Pierpont EI, Nascene DR, Shanley R, et al. Neurocognitive benchmarks following transplant for emerging cerebral adrenoleukodystrophy. Neurology 2020;95:e591-600 CrossRef Medline

14. Vogel BH, Bradley SE, Adams DJ, et al. Newborn screening for Xlinked adrenoleukodystrophy in New York State: diagnostic protocol, surveillance protocol and treatment guidelines. Mol Genet Metab 2015;114:599-603 CrossRef Medline

15. Loes DJ, Hite S, Moser H, et al. Adrenoleukodystrophy: a scoring method for brain MR observations. AJNR Am J Neuroradiol 1994;15:1761-66 Medline

16. Musolino PL, Rapalino O, Caruso P, et al. Hypoperfusion predicts lesion progression in cerebral X-linked adrenoleukodystrophy. Brain 2012;135:2676-83 CrossRef Medline

17. Pierpont EI, Eisengart JB, Shanley R, et al. Neurocognitive trajectory of boys who received a hematopoietic stem cell transplant at an early stage of childhood cerebral adrenoleukodystrophy. JAMA Neurol 2017;74:710-17 CrossRef Medline

18. Moser AB, Kreiter N, Bezman L, et al. Plasma very long chain fatty acids in 3,000 peroxisome disease patients and 29,000 controls. Ann Neurol 1999;45:100-10 CrossRef Medline

19. Eichler F, Duncan C, Musolino PL, et al. Hematopoietic stem-cell gene therapy for cerebral adrenoleukodystrophy. $N$ Engl J Med 2017;377:1630-38 CrossRef Medline

20. Kikinis R, Pieper SD, Vosburgh Kg 3D. Slicer a platform for subjectspecific image analysis, visualization, and clinical support. In: Jolesz FA, ed. Intraoperative Imaging and Image-Guided Therapy. Springer-Verlag New York; 2014:277-89

21. Egger J, Kapur T, Fedorov A, et al. GBM volumetry using the 3D slicer medical image computing platform. Sci Rep 2013;3:1364-67 CrossRef Medline

22. Mallack EJ, Turk BR, Yan H, et al. MRI surveillance of boys with Xlinked adrenoleukodystrophy identified by newborn screening: metaanalysis and consensus guidelines. J Inherit Metab Dis 2021;44:728-39 CrossRef Medline

23. Shapiro E, Krivit W, Lockman L, et al. Long-term effect of bonemarrow transplantation for childhood-onset cerebral X-linked adrenoleukodystrophy. Lancet 2000;356:713-18 CrossRef Medline

24. Lebel C, Beaulieu C. Longitudinal development of human brain wiring continues from childhood into adulthood. J Neurosci 2011;31:10937-47 CrossRef Medline

25. Aubert-Broche B, Fonov V, Narayanan S, et al. Canadian Pediatric Demyelinating Disease Network. Onset of multiple sclerosis before adulthood leads to failure of age-expected brain growth. Neurology 2014;83:2140-46 CrossRef Medline

26. Tillema JM, Derks MG, Pouwels PJ, et al. Volumetric MRI data correlate to disease severity in metachromatic leukodystrophy. Ann Clin Transl Neurol 2015;2:932-40 CrossRef Medline

27. Sicotte NL, Voskuhl RR, Bouvier S, et al. Comparison of multiple sclerosis lesions at 1.5 and 3.0 Tesla. Invest Radiol 2003;38:423-27 CrossRef Medline

28. Kothari RU, Brott T, Broderick JP, et al. The ABCs of measuring intracerebral hemorrhage volumes. Stroke 1996;27:1304-05 CrossRef Medline

29. Chang K, Beers AL, Bai HX, et al. Automatic assessment of glioma burden: a deep learning algorithm for fully automated volumetric and bidimensional measurement. Neuro Oncol 2019;21:1412-22 CrossRef Medline

30. Wattjes MP, Rovira À, Miller D, et al. MAGNIMS study group. Evidence-based guidelines: MAGNIMS consensus guidelines on the use of MRI in multiple sclerosis: establishing disease prognosis and monitoring patients. Nat Rev Neurol 2015;11:597-606 CrossRef Medline 